\title{
Conf-9305377--3
}

DOE/ER/14079-34

CWP-154P

April 1994

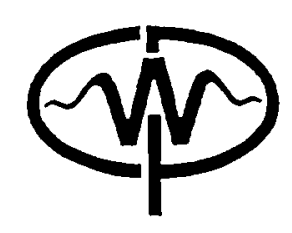

\section{D Depth Migration in Transversely Isotropic Media Using Explicit Operators}

by

\author{
Omar Uzcategui
}

This paper was presented at the CWP Annual Project Review Meeting, May 11-14, 1993, Breckenridge, Colorado. It has also been submitted to GEOPHYSICS.

\section{DISCLAIMER}

This report was prepared as an account of work sponsored by an agency of the United States Government. Neither the United States Government nor any agency thereof, nor any of their employees, makes any warranty, express or implied, or assumes any legal liability or responsibility for the accuracy, completeness, or usefulness of any information, apparatus, product, or process disclosed, or represents that its use would not infringe privately owned rights. Reference herein to any specific commercial product, process, or service by trade name, trademark, manufacturer, or otherwise does not necessarily constitute or imply its endorsement, recommendation, or favoring by the United States Government or any agency thereof. The views and opinions of authors expressed herein do not necessarily state or reflect those of the United States Government or any agency thereof.

\section{Center for Wave Phenomena \\ Colorado School of Mines \\ Golden, Colorado 80401}

(303)273-3557

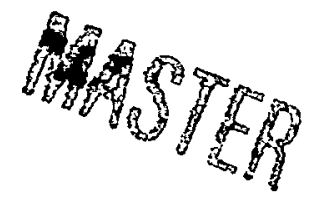




\section{DISCLAIMER}

Portions of this document may be illegible in electronic image products. Images are produced from the best available original document. 


\title{
2D depth migration in transversely isotropic media using explicit operators
}

\author{
Omar Uzcategui
}

\begin{abstract}
Stable, explicit depth-extrapolation filters can be used to propagate plane waves corresponding to the $\mathrm{qP}$ and $\mathrm{qSV}$ (quasi-P and quasi-SV propagation) modes for transversely isotropic (TI) media. Here, I discuss and compare results of two different methods for obtaining the filters for TI media with a vertical axis of symmetry (VTI). The first, a modified Taylor series method, is used to calculate the $N$-coefficients of a finite-length filter such that the Taylor expansion around vertical propagation matches the spatial Fourier transform of the downward-continuation operator for VTI media. Second, a least-squares method is used to calculate the filter coefficients such that the amplitude and phase departures from the ideal response of the downwardcontinuation operator for VTI media are minimized over a range of frequencies and propagation angles. In both methods, the amplitude response of the filter is forced to be less than unity in the evanescent region in order to achieve stability.

In general, as exemplified in all the cases studied here, the constrained leastsquares method produced filters with accurate wavefield extrapolation for a wider range of propagation angles than that obtained for the modified Taylor series method. In both methods, the maximum angle that can be accurately propagated depends on the ratio of frequency to vertical phase velocity $\left(f / V_{v}\right)$, and on the length of the filter. However, for a fixed filter length and for a given ratio $f / V_{v}$, the maximum angle propagated with accuracy depends on the elastic constants of the medium. The accuracy of the filters degrades as the degree of anisotropy becomes more extreme.
\end{abstract}

\section{INTRODUCTION}

The assumption of elastic isotropy has been useful in petroleum geophysics; however, many sedimentary rocks are found from experiment to be anisotropic. Theoretical studies (Postma, 1955; Levin, 1979), velocity determinations in the laboratory (Thomsen, 1986), and field studies (Banik, 1984; White et al., 1983; Crampin et al., 1984; Ball, 1993) have shown that many sedimentary rocks are anisotropic. In particular, transversely isotropic (TI) media have been observed in several studies ( Jones and Wang, 1981; Ball, 1993), and it is expected that media formed through rapid 
cyclical sedimentation patterns will be transversely isotropic or exhibit more complex anisotropy (Backus, 1962).

Clearly, since seismic imaging requires use of accurate migration velocity, anisotropy can play an important role in the positioning of geological boundaries on migrated images. Larner and Cohen (1993) and Alkhalifah and Larner (Alkhalifah, T., and Larner, K., 1993, Migration error in transversely isotropic media: Center for Wave Phenomena Annual Report, CWP-133, Colorado School of Mines), have found that large position errors arise for steep reflectors when transverse isotropy is ignored in post-stack migration. Isotropic migration algorithms applied to physical modeling data in TI media (Martin et al., 1992) produced mis-location of plane reflectors and a complete loss of steep structures.

Recognizing the importance of taking anisotropy into account, several authors have recently developed migration algorithms for anisotropic media. Uren et al. (1990) evaluated the performance of an two-dimensional (2D) $w-k$ depth migration algorithm in TI media. Sena and Toksöz (1993) developed a 2D Kirchhoff pre-stack depth migration algorithm using a Green's tensor representation for weakly TI media, and Alkhalifah (Alkhalifah, T., 1993, Gaussian beam migration for anisotropic media: Center for Wave Phenomena Annual Report, CWP-128, Colorado School of Mines) used a Gaussian beam algorithm for post-stack migration in general 2D anisotropic media. Kitchenside (1993) developed both implicit and explicit schemes for $\omega-x$ downward-continuation in 2D TI media. The explicit scheme involves the spatial inverse Fourier transform of the discrete downward-continuation operator for TI media in the $\omega-k$ domain; the resulting operator is then truncated and tapered to reduce Gibbs-phenomenon. However, as pointed out by Nautiyal et al. (1993), long operators or a strong tapering, with consequent loss of accuracy in the propagation region, are needed to get stable filters by using this method.

Explicit filters (Holberg, 1988; Hale, 1991a) have proved to be a useful tool in the extrapolation of seismic wavefields for depth migration in isotropic media. Here, I extend their use to TI media. Explicit filtering is attractive because it can be implemented efficiently on pipelined and parallel computers. In addition, explicit methods can be easily extended for 3D depth migration in isotropic, heterogenous media (Hale, 1991b). For TI media, explicit depth-extrapolation filters can be obtained by following the same steps as in the isotropic case, but with special considerations for the anisotropic nature of the phase velocity.

The modified Taylor series method used by Hale (1991a) for isotropic media can be adapted for transversely isotropic media with vertical axis of symmetry (VTI), but now taking into account that the horizontal wavenumber and the phase velocity are functions of the propagation angle. The basic idea is to find a filter in frequencymidpoint $(\omega-x)$ space such that its spatial Fourier transform matches (in the propagation region) that of the downward continuation operator for TI media. To ensure attenuation of the evanescent energy, some degrees of freedom in the filter are used to force amplitude values to be less than unity in the evanescent region. 
In an alternative approach, Holberg (1988) obtained explicit depth-extrapolation filters for isotropic media by minimizing the squared error of amplitude and phase of the filter in a given range of propagation angles with the vertical $\left(0 \leq|\theta| \leq \theta_{\max }\right)$. For VTI media I follow the same approach, but now take into account the theoretical expression of the downward-continuation operator for VTI media. Much as was done by Holberg, to get stability for angles greater than $\theta_{\max }$ and in the evanescent region, I introduce a penalty function that grows for amplitude values of the filter greater than unity. I then use a conjugate-gradient algorithm to minimize the resulting function.

\section{FILTER DESIGN}

The theoretical downward-continuation operator for VTI media can be obtained from the equations of motion for elastic plane waves propagating in the vertical $(x, z)$ plane (Kitchenside, 1991). The match with the theoretical operator is done only in the propagation region; the filter must attenuate the energy in the evanescent region. For a particular frequency $\omega$ in the propagation region, the spatial Fourier transform of the filter $W\left(k_{x}, \omega\right)$ must approximate

$$
D\left[k_{x}(\theta), \omega\right]=\exp \left\{i \frac{\Delta z}{\Delta x}\left[\left(\frac{\omega \Delta x}{V_{p}(\theta)}\right)^{2}-k_{x}^{2}(\theta)\right]^{\frac{1}{2}}\right\},
$$

where $D\left[k_{x}(\theta), \omega\right]$ is the exact downward-continuation operator for the medium, $\Delta z$ and $\Delta x$ are the vertical and horizontal sampling intervals, and $V_{p}(\theta)$ and $k_{x}(\theta)$ are the phase velocity and the horizontal wavenumber for a given propagation mode (qP or qSV), evaluated at the phase angle $\theta . k_{x}(\theta)$ is related to the phase velocity by

$$
\frac{k_{x}(\theta)}{\Delta x}=\frac{\omega \sin (\theta)}{V_{p}(\theta)} .
$$

$W\left(k_{x}, \omega\right)$ can be written as

$$
W\left(k_{x}, \omega\right)=\sum_{l=0}^{(N-1) / 2}\left(2-\delta_{l 0}\right) h_{l}(\omega) \cos \left(k_{x} l\right),
$$

where $\delta_{10}$ is the Kronecker delta function, $h_{l}(\omega)$ are complex coefficients of the filter, and $N$ is the number of coefficients. Because $W\left(k_{x}, \omega\right)$ is symmetric in $k_{x}$, only $(N+1) / 2$ different coefficients $h_{l}(\omega)$ are needed to determine the response of an $\mathrm{N}$-length filter.

In the modified Taylor series method (Hale, 1991a), the filter coefficients $h_{l}(\omega)$ are obtained by matching the filter's Taylor expansion around $\theta=0$ with that of the downward-continuation operator. In particular, because the filter is symmetric and we want this filter to be exact for vertical propagation, we would like to match 
the first $(N+1) / 2$ even derivatives at $\theta=0$. However, to obtain attenuation in the evanescent region, we must try to match fewer than $(N+1) / 2$ derivatives and let the remaining degrees of freedom in the filter be used to ensure that the amplitude of the filter is less than unity in that region $\left(\left|W\left(k_{x}, \omega\right)\right| \leq 1\right)$. Following Hale (1991a), the filter coefficients are represented as a sum of $M$ weighted basis functions:

$$
h_{l}(\omega)=\sum_{m=0}^{M-1} c_{m}(\omega) b_{m l},
$$

where the basis function $b_{m l}$ is given by

$$
b_{m l}=\left(2-\delta_{m 0}\right) \cos \left(\frac{2 \pi m l}{N}\right) .
$$

The problem is now to determine the $M$ complex weights $c_{m}(\omega)$. To achieve stability, the number $M$ of weights must be less than the number $(N+1) / 2$ of filter coefficients, so only the first $M$ derivatives of the Fourier transform of the filter are matched with those of the downward-continuation operator for VTI media. The remaining $(N+1) / 2-M$ degrees of freedom are used to ensure stability.

The Fourier transform of the extrapolation filter will be now

$$
W\left(k_{x}, \omega\right)=\sum_{m=0}^{M-1} c_{m}(\omega) B_{m}\left[k_{x}(\theta)\right] \simeq D\left[k_{x}(\theta), \omega\right]
$$

where $B_{m}\left[k_{x}(\theta)\right]$ is given by

$$
B_{m}\left[k_{x}(\theta)\right]=\left(2-\delta_{m 0}\right) \sum_{l=0}^{(N-1) / 2}\left(2-\delta_{l 0}\right) \cos \left(\frac{2 \pi m l}{N}\right) \cos \left[k_{x}(\theta) l\right] .
$$

The main difference between this Fourier transform of the basis function $B_{m}\left[k_{x}(\theta)\right]$ and that given by Hale (1991a) for isotropic media is that in this case (VTI media) the horizontal wavenumber $k_{x}$ also depends on $\theta$ through $V_{p}(\theta)$ [see equation (2)]. In equation (6), $M$ terms in the Taylor series expansion around $\theta=0$ for the filter $W\left(k_{x}, \omega\right)$ are matched with those terms corresponding to the Taylor series expansion of the downward continuation operator $D\left(k_{x}(\theta, \omega)\right.$. Whereas Hale's Taylor series expansions were in powers of $k_{x}$, the expansions here are in powers of $\theta$

$$
\begin{aligned}
\left.W\left[k_{x}(\theta), \omega\right)\right] \simeq & \sum_{m=0}^{M-1} c_{m}(\omega)\left[B_{m}(0)+\frac{B_{m}^{2}(0) \theta^{2}}{2 !}+\frac{B_{m}^{4}(0) \theta^{4}}{4 !}+\ldots \frac{B_{m}^{2 M}(0) \theta^{2 M}}{(2 M) !}\right] \\
& D\left[k_{x}(\theta), \omega\right] \simeq D(0)+\frac{D^{2}(0) \theta^{2}}{2 !}+\frac{D^{4}(0) \theta^{4}}{4 !}+\ldots \frac{D^{2 M}(0) \theta^{2 M}}{(2 M) !}
\end{aligned}
$$


where $D^{(2 l)}(0)$ and $B_{m}^{(2 l)}(0)$ are the $2 l^{\text {th }}$ derivatives of the downward-continuation operator and basis functions, respectively, evaluated at $\theta=0$. Matching of these Taylor expansions gives a linear system of equations for the coefficients $c_{m}(\omega)$

$$
\begin{array}{r}
\sum_{m=0}^{M-1} c_{m}(\omega) B_{m}^{(2 l)}(0)=D^{(2 l)}(0) \quad l=0,1, \ldots M \\
B_{m}^{(2 l)}(0)=\left(2-\delta_{m 0}\right) \sum_{n=0}^{(N-1) / 2} \cos \left(\frac{2 \pi m n}{N}\right) \cos \left[n k_{x}(\theta)\right]^{(2 l)}(0),
\end{array}
$$

where $\cos \left[n k_{x}(\theta)\right]^{(2 l)}(0)$ is the $2 l^{\text {th }}$ derivative of $\cos \left[n k_{x}(\theta)\right]$ evaluated at $\theta=0$. For isotropic media $\cos \left[n k_{x}(\theta)\right]^{(2 l)}(0)=(-1)^{2 l-1} \sum_{i=1}^{l} a_{i}\left[n \omega / V_{p}(0)\right]^{2 i}$, where the $a_{i}$ are constant coefficients. For VTI media, the cosine derivatives can still be expressed as powers of $n \omega / V_{p}(0)$, but the coefficients $a_{i}$ are now functions of the phase-velocity derivatives evaluated at $\theta=0$. The system of equations [8] and [9] is solved for $c_{m}(\omega)$, and equation (4) is used to calculate the final filter coefficients $h_{l}(\omega)$.

For the least-squares method, the match of the Fourier transform of the filter with the downward-extrapolation operator is done over a range of phase angles $(\theta=$ $\left.0, . ., \theta_{\max }\right)$. Following Holberg (1988), we do the matching by minimizing the sum of the square errors in the amplitude and phase response of the filter,

$$
J(\omega)=\int_{\theta=0}^{\theta=\theta_{\max }}\left(A E^{2}+P E^{2}\right) d k_{x}
$$

subject to the constraints

$$
\left|W\left(k_{x}, \omega\right)\right|<1.0 \text { for }\left|k_{x}\right|>\frac{\omega \sin \left(\theta_{\max }\right)}{V_{p}\left(\theta_{\max }\right)} .
$$

Here, $A E$ is the amplitude error of the filter, $A E \equiv 1.0-\left|W\left(k_{x}, \omega\right)\right|$, and $P E$ is the phase error of the filter, $P E \equiv k_{z} \Delta z-\tan ^{-1} \frac{\operatorname{Im}\left(W\left(k_{x}, \omega\right)\right)}{\operatorname{Re}\left(W\left(k_{x}, \omega\right)\right)}$.

Constraint (11) was incorporated into the least-squares problem (10) by adding to the error function $J(\omega)$ a penalty function that is proportional to the square of the amplitude error when the amplitude of the filter exceeds unity. I then use a conjugate gradient algorithm for the minimization of the non-linear least-squares problem.

\section{FILTER ACCURACY FOR A SINGLE FREQUENCY}

\section{Taylor series method}

For the Taylor series method we can see from equation (8) that the degree to which the filter would approach the downward-continuation operator depends on the number $M$ of terms used in the Taylor expansion. Long filters allow use of more terms, so 


\begin{tabular}{|c|c|c|c|c|}
\hline Medium & $\begin{array}{c}\alpha_{0} \\
\mathrm{~km} / \mathrm{s}\end{array}$ & $\begin{array}{c}\beta_{0} \\
\mathrm{~km} / \mathrm{s}\end{array}$ & $\epsilon$ & $\delta$ \\
\hline Berea sandstone & 4.206 & 2.664 & 0.002 & 0.020 \\
\hline Taylor sandstone & 3.368 & 1.829 & 0.110 & -0.035 \\
\hline Mesaverde clayshale & 3.794 & 2.074 & 0.189 & 0.204 \\
\hline weathered gypsum & 1.911 & 0.795 & 1.161 & -0.140 \\
\hline
\end{tabular}

Table 1. Vertical phase velocities (P-wave $\alpha_{0}$ and S-wave $\beta_{0}$ ) and Thomsen anisotropy parameters $(\epsilon$ and $\delta$ ) for several VTI media.

they better approximate the $D\left(k_{x}, \omega\right)$ operator than do short filters. However, for VTI media the downward-continuation operator is also a function of the phase velocity, whose variation with respect to the propagation angle $\theta$ changes from one medium to another. For media with smooth variation of $V_{p}(\theta)$ with respect to $\theta$, the Taylor expansion of $V_{p}(\theta)$ would be a good representation of $V_{p}(\theta)$ itself, and only a few terms in (8) would be used. For strongly anisotropic media with large variations of $V_{p}(\theta)$, more terms would be needed in equation (8).

Figure 1 shows amplitude spectra of filters in the propagation region $\left(0^{\circ} \leq \theta \leq\right.$ $90^{\circ}$ ) obtained using the Taylor series method, for several VTI media and for both propagation modes ( $\mathrm{qP}$ and $\mathrm{qSV}$ ). The vertical phase velocities and anisotropy parameters (Thomsen, 1986) for these media are given in Table 1. The amplitude scale displays only the region close to unity to show the stability in the propagation region. The number of terms in the expansion was $N=39, \Delta z / \Delta x=1$, and for all the cases shown, the ratio $f \Delta x / V_{p}(0)$ (normalized frequency, in cycles) is constant and equal to 0.25 . This ratio was kept constant because the downward-continuation operator is basically a function of $f \Delta x / V_{p}(\theta)$. If I had fixed $f$, the ratio $f \Delta x / V_{p}(0)$ would change with $V_{p}(0)$ for the different media, and thus the comparison would not be fair.

According to Thomsen (1986), a medium can be considered as weakly anisotropic if the values of the anisotropy parameters $\epsilon$ and $\delta$ are small with respect to unity. On this basis, the Berea sandstone can be considered as weakly anisotropic, the Taylor sandstone as moderately anisotropic, the weathered gypsum as highly anisotropic (not likely to be encountered), and the Mesaverde clayshale as an elliptically anisotropic material $(\epsilon \simeq \delta)$. From Figure 1 and for the $q P$ propagation mode, we see that for the same length of the filter and ratio $f \Delta x / V_{p}(0)$, the filters can propagate accurately (amplitude error less than 0.1 percent) to a maximum angle of some 45 degrees in the Taylor sandstone and Mesaverde clayshale, 55 degrees in the Berea sandstone, and only 25 degrees in the weathered gypsum. For the Berea sandstone, the maximum accurate propagation angle (55 degrees), is close to that found by Hale (1991a) for isotropic media. Note that for the qP mode the error curves in Figure 1 for the Mesaverde clayshale and Taylor sandstone are similar. Although the values of $\epsilon$ and $\delta$ for Mesaverde clayshale are higher than those for Taylor sandstone, the shape of 
the phase-velocity curve for $\epsilon \simeq \delta$ (Mesaverde clayshale) is more simple. As a result, the Mesaverde clayshale behaves as a moderately anisotropic material (similar to the Taylor sandstone) for the $\mathrm{qP}$ propagation mode. Behavior similar to that for all of the amplitude curves in Figures 1 through 3 holds for phase errors as well (not shown here).

For the qSV propagation mode, the filters can again propagate accurately to about 55 degrees in the Berea sandstone, and, interestingly, the filter for Mesaverde clayshale is more accurate for the qSV propagation than for qP propagation. The Mesaverde clayshale behaves as an isotropic material for the qSV propagation mode (there is no phase-velocity dependence on $\theta$ for $\epsilon \simeq \delta$ ). For Taylor sandstone, accuracy is reduced somewhat to a maximum angle of 40 degrees, and, for the weathered gypsum, accurate results are confined to propagation angles less than only 15 degrees.

Figure 2 shows amplitude spectra of the filters in Figure 1, now as a function of normalized wavenumber (in cycles) rather than dip; 0.5 cycles corresponds to Nyquist wavenumber $(\pi / \Delta x)$. Also, the amplitude scale has been increased to show attenuation in the evanescent region (normalized wavenumbers greater than 0.25 ). The small amplitudes in the evanescent region show that the extrapolators are clearly stable in that region.

\section{Least-squares method}

The accuracy of filters designed by the least-squares method will also depend on the number of coefficients used, but now, we match the filter response with the exact downward continuation operator and not with its Taylor series expansion, as we did in the Taylor series method. This approach will avoid the progressively more poor fit of the theoretical operator by the Taylor series expansion for increasing propagation angles. However, the amplitude spectrum of a filter designed by least-squares methods is found to have an oscillatory character, as seen in Figure 3, with amplitude values greater than unity for some wavenumbers in the propagation region. As demonstrated by Holberg (1988), the magnitude of these oscillations is reduced by restricting the range of propagation angles for which the fit is done. This introduces a new parameter (absent in the Taylor series method) in the design of the filter- the maximum design angle $\left[\theta_{\max }\right.$ in equation (10)].

Figure 3 shows amplitude spectra as a function of dip for the same VTI media shown in Figure 1 and for filters obtained using the least-squares method. Note the ripples in amplitude in the propagation region. Although some of these amplitude values are greater than unity, they do not exceed 1.001, which guarantees that application of these filters for about one-thousand steps of downward extrapolation would magnify amplitudes by no more than $e=2.71$. [This is the same criterion as that used by Hale (1991a).] Also, from Figure 3 we see that the maximum accurate propagation angles obtained by the least-squares method are, for all media studied, greater than those obtained by the modified Taylor series method.

For a given length of filter, there exists a maximum design angle $\left(\theta_{\max }\right)$ in the 
least-squares method beyond which accuracy and stability are lost. This maximum allowed design angle increases somewhat as filter length increases. Here, I define an accurate and stable filter as one having a maximum absolute amplitude error of no more than 0.001 in the region $\theta \leq \theta_{\max }$, and amplitude values less than unity for $\theta_{\max }<\theta \leq 90^{\circ}$ and in the evanescent region. An N-coefficient filter cannot achieve both goals (accuracy and stability) for $\theta_{\max }$ close to $90^{\circ}$ because not enough degrees of freedom are available to satisfy both conditions. The maximum design angles in Figure 3 were obtained after several tests with differents $\theta_{\max }$, beginning with a high initial value of $\theta_{\max }$ and reducing this value until an accurate and stable filter was found. For the four media studied, the determined maximum design angles were as follows. $\mathrm{qP}$ mode: 70 degrees for the Berea sandstone, 65 degrees for the Taylor sandstone and Mesaverde clayshale, and only 30 degrees for the weathered gypsum. qSV mode: 70 degrees for the Berea sandstone and Mesaverde clayshale, 60 degrees for the Taylor sandstone, and only 20 degrees for the weathered gypsum.

\section{ACCURACY FOR A RANGE OF FREQUENCIES}

In application to broad band data, depth-extrapolation filters must be designed to propagate a wide range of frequencies. Figure 4 shows the amplitude and phase errors for the Taylor sandstone using a 39-coefficient filter based on the modified Taylor series method. The errors are shown as a function of normalized frequency $\tilde{f}=f \Delta x / V_{p}(0)$ and propagation angle. The contour correspond to an amplitude error of $1 / 1000$. The amplitude error was calculated as the difference $1.0-|W(\tilde{f}, \theta)|$, so positive values in the error indicate amplitude values in the filter less than unity. An amplitude error of $1 / 1000$ corresponds to a filter amplitude of 0.999 ; after 1000 steps of downward extrapolation with this error, the amplitude of the initial waveform extrapolated will be $(0.999)^{1000} \simeq 0.4$ times the initial amplitude. The phase error contour corresponds to an error of $\pi / 1000$ radians; after 1000 steps of downward extrapolation, the accumulated error in phase will be one-half cycle ( $\pi$ radians). Figure 4 thus shows that the filter for Taylor sandstone will attenuate waves propagating at about 45 degrees by a factor of 0.4 , and with one-half cycle of phase error after 1000 steps of downward extrapolation.

Figure 5 shows the corresponding errors for a 39-coefficient filter and the Taylor sandstone, but now using the least-squares method. After 1000 steps of downward extrapolation, seismic waves propagating at more than 60 degrees will accumulate error that is comparable to errors for waves propagating at only 45 degrees in Figure 4. Thus, for the same number of filter coefficients, more than 15 degrees of reflector dip has been gained through use of the least-squares approach.

Figure 6 compares amplitude errors of a 39-coefficient filter for the $\mathrm{qP}$ mode in the weathered gypsum using the Taylor series and least-squares methods. The design angle used in the least-squares method was 30 degrees. Note the small increase ( $\simeq 5$ degrees) in the range of angles for accurate propagation when the least-squares method is used. Figure 7 shows the corresponding errors for the qSV propagation 
mode in the Taylor sandstone. The design angle used in the least-squares method was 60 degrees. Comparing with Figure 4, we see that for a given medium (Taylor sandstone), the range of angles for accurate propagation is better for the $\mathrm{qP}$ than for the qSV mode. Note in Figure 7 the irregular error in amplitude and phase produced by the Taylor-series filter; however, for the least-squares filter, the error is uniform for angles less than the design angle ( $\theta_{\max }=60$ degrees).

In Figures 4 through 7 , note that the accuracy, as measured by the contours shown, is relatively insensitive to frequency for most of the frequency range of interest. The same behavior holds for amplitude and phase errors for the Mesaverde clayshale and Berea sandstone (not shown here). For those media, the results shown in Figures 1 through 3, for a single frequency, are representative of those over the range of frequencies of interest.

\section{MIGRATION IMPULSE RESPONSES IN HOMOGENOUS MEDIA}

The performance of the operators can perhaps best be exemplified through study of migration impulse responses. The same $\omega-x$ domain migration program used by Hale (1991a) for isotropic media was used here to calculate the migration impulse responses for the $\mathrm{qP}$ and $\mathrm{qSV}$ propagation modes. This same program can be used because in migration by explicit filtering, the downward-continuation process is performed by space-variant convolution of the data with the explicit filters (Holberg, 1988). The convolution process is the same for both isotropic and VTI media; we need only change the filter coefficients. In addition, the number of filters needed and their length (with the dip restrictions discussed before) are equal in both media; therefore, the computational cost of migration is exactly the same.

Figure 8 shows qP impulse responses for the Taylor sandstone using the modified Taylor series method (a) and the least-squares method (b). In both tests, spatial sampling intervals $\Delta x=\Delta z=10 \mathrm{~m}$, and the time sampling interval $\Delta t=10 \mathrm{~ms}$; therefore, the Nyquist frequency is $50 \mathrm{~Hz}$. For these parameters, the normalized frequency $\left(\tilde{f}=f \Delta x / V_{p}(0)\right)$ varies from 0 to 0.3 cycles. The trajectory of the theoretical impulse response (shown in the figures as a thin solid line) was calculated using the theoretical group velocity and ray angle (Thomsen, 1986) for this medium. Note that the large dips are more severely attenuated by the modified Taylor series filter than by the least-squares filter. Although steep events (corresponding to high propagation angles) are attenuated for both filters, the fit with the theoretical impulse response is good for a wide range of angles.

Figure 9 shows qP impulse responses for the highly anisotropic weathered gypsum, using the modified Taylor series method (a) and the least-squares method (b). The largest dip present in this response is lower than that obtained for the $\mathrm{qP}$ Taylor sandstone filter (compare with Figure 8). This basically reflects the behavior of the amplitude error shown in Figure 6, where wavefield components with dips greater than 30 degrees are propagated with large positive errors corresponding to amplitude values less than unity. Therefore, these components will be attenuated. 
Figure 10 shows qSV impulses for the Taylor sandstone (a) and Mesaverde clayshale (b), respectively. Both responses were calculated using 39-coefficient filters derived by the more accurate least-squares method. Note the attenuation at large dips for the Taylor sandstone. However, for the Mesaverde clayshale, steep events can be propagated accurately. Given the same number of coefficients for both media, more accurate propagation of the qSV wave is obtained for the elliptical material.

\section{INHOMOGENOUS MEDIA}

Explicit extrapolators in isotropic media can easily handle lateral velocity variations. As pointed out by Holberg (1988) and Hale (1991a), lateral velocity variations are handled by lateral variable extrapolators. A table of extrapolators is calculated for a typical range of normalized frequencies $f \Delta x / V_{p}$; then, during the depth-extrapolation process, from one depth to the next and for a constant frequency $f$, lateral velocity variations are handled by using different filters. Thus, for constant spatial sampling intervals $\Delta x$ and $\Delta z$, a filter calculated to propagate the normalized frequency $f_{1} \Delta x / V_{1 p}$ can also be used to propagate any normalized frequency $f_{2} \Delta x / V_{2 p}$ such that $f_{1} / V_{1 p}=f_{2} / V_{2 p}$.

In VTI media the problem is more complex because the qP and qSV phasevelocity, and, therefore, the downward-continuation operator are functions of four variables (Thomsen's parameters). Therefore, it might seem that we need a different extrapolator table for each set of Thomsen's parameters. However, a careful look at the phase-velocity equations for VTI media [equations (10a) and (10b) in Thomsen, 1986] reveals that the dependence on vertical velocity for $\mathrm{qP}$ and qSV is just a multiplicative factor. In addition, as suggested by Tsvankin (Tsvankin, I., 1993, Analytic description of dip moveout in anisotropic media: Center for Wave Phenomena Annual Report, CWP, Colorado School of Mines), the ratio of vertical velocities, $\alpha_{0} / \beta_{0}$, does not have a significant influence on the $\mathrm{qP}$ phase-velocity, for typical values of $\epsilon$ and $\delta$ found in sedimentary basins (the same is not true for the qSV phase-velocity). Therefore, for the $\mathrm{qP}$ propagation mode, only one extrapolator table is needed for each constant pair of $\epsilon$ and $\delta$ values required to characterize a medium, no matter how the vertical phase velocities $\left(\alpha_{0}, \beta_{0}\right)$ change laterally or in depth.

Additional simplifications are obtained for factorized transversely isotropic (FTI) media. FTI media are characterized by having all ratios among the elastic coefficients independent of position (Červený, 1989), e.g., $\delta$ and $\epsilon$ are independent of position. For such media, the dependence of phase velocity on direction is therefore also independent of position. As a result, for this kind of medium, only one table of depth-extrapolators is needed.

Figure 11 shows a model containing horizontal reflectors and segments with dip ranging from 30 to 90 degrees. The medium has the elastic properties of Mesaverde clayshale, with a $P$-wave vertical velocity $\alpha_{0}(x, z)=3.794+0.5 x+0.5 z \mathrm{~km} / \mathrm{s}$, where $x$ and $z$ are in $\mathrm{km}$. Figure 12a shows the $\mathrm{qP}$ zero-offset section correspondent to this model and calculated using a ray tracing program for FTI media (Alkalifah, T., 1993, 
Efficient synthetic seismograms for transversely isotropic media with constant velocity gradient: Center for Wave Phenomena Annual Report, CWP-128, Colorado School of Mines). Figure 12b shows the depth-migration obtained using Hale's (1991a) $w-x$ depth-migration program, with just one least-square $\mathrm{qP}$ table of depth-extrapolators. The maximum design angle in the LS method was 60 degrees; therefore, only reflectors with dip up to 60 degrees are recovered. The 75 -degree reflector is attenuated and mis-placed due to the amplitude and phase errors of the filter for dip greater than 60 degrees. The 90-degree reflector is completely lost.

For the depth-migration calculation (Figure 12b), 501 common midpoints, 301 time samples, and 401 depth extrapolation steps were used. The total CPU time on the IBM RS/6000 Model 530 workstation was 20.46 minutes, the same as for an isotropic migration for a medium with the same heterogeneity.

\section{CONCLUSION}

Explicit 2D depth-extrapolation filters for VTI media can be derived either by a generalization of the modified Taylor series method or by a least-squares method initially developed for isotropic media. Also, just as for isotropic media, these 2D extrapolators for VTI media can be used in the McClellan transformation (Hale, 1991b) to do 3D depth migration.

The results shown here are limited to just TI media with vertical axis of symmetry. A similar approach with tilted axis of symmetry can be developed if the theoretical downward-continuation operator for such media is used in the equations developed here. Apparently, the generalization requires only that the modifications appropriate for tilted axis of symmetry be made to the phase-velocity during the operator design. However, the extension to $3 \mathrm{D}$ is not straightforward due to a loss of symmetry in the horizontal plane.

Qualitatively, the results in this paper show that the accuracy of the extrapolators (for a constant filter-length and normalized frequency) is a function of the elastic properties in the medium. In particular, for non-elliptical anisotropy, more-accurate filters are obtained for weakly than for strongly anisotropic media. Because the phase-velocity dependence on propagation angle is larger for strongly anisotropic than that for weakly anisotropic media, weakly anisotropic media need fewer filter coefficients than do strongly anisotropic media to produce a good representation of the downward-continuation operator. Further analysis is necessary to study the role of the anisotropy parameters $(\epsilon$ and $\delta$ ) in governing the accuracy of the extrapolators. Also, because there is no phase-velocity dependence on $\theta$ for elliptical media $(\epsilon \simeq \delta)$ in the qSV mode, filters for that mode have accuracy comparable to that for isotropic media. For the $\mathrm{qP}$ mode in media with elliptical anisotropy the accuracy is relatively insensitive to the degree of anisotropy.

The examples presented also show that the constrained least-squares method produces filters with accurate extrapolation for a wider range of propagation angles than 
that obtained for the modified Taylor series method. Again, the accuracy in both methods degrades as the degree of anisotropy becomes more extreme. For weakly and moderately anisotropic media, the accuracy of the filters in doing downward extrapolation is comparable to that obtained by Hale (1991a) and by Holberg (1988) for isotropic media. Some alternative approach to improve the accuracy of the operators for strongly anisotropic media might be suggested once a quantitative analysis of the influence of $\epsilon$ and $\delta$ on the accuracy of the filters is performed.

\section{ACKNOWLEDGMENTS}

The author thanks INTEVEP, Venezuela, for its financial support. Thanks also to professors Ken Larner and Ilya Tsvankin for their critical review of this paper. This project is supported by the members of the Consortium Project on Seismic Inverse Methods for Complex Structures at the Center for Wave Phenomena, Colorado School of Mines and the U.S. Department of Energy, Grant number DE-FG02-89ER14079 (this support does not constitute an endorsement by DOE of the views expressed in this paper).

\section{REFERENCES}

Backus, G. E., 1962, Long-wave elastic anisotropy produced by horizontal layering: J. Geophys. Res., 67, 4427-4440.

Ball, G. J., 1993, Anisotropic 3D prestack depth migration, offshore Zaire: 63th Ann. Internat. Mtg., Soc. Expl. Geophys., Expanded Abstracts, 843-846.

Banik, N. C., 1984, Velocity anisotropy of shales and depth estimation in the North Sea basin: Geophysics, 49, 1411-1419.

Červený, V., 1989, Ray tracing in factorized anisotropic inhomogenous media: Geophys. J. Int., 94, 575-580.

Crampin, S., Chesnokov, E. M., and Hipkin, R. A., 1984, Seismic anisotropy- the state of the art: Geophys. J. Roy. Astr. Soc., 76, 1-16.

Hale, I. D., 1991a, Stable explicit depth extrapolation of seismic wavefields: Geophysics, 56, 1770-1777.

Hale, I. D., 1991b, 3-D depth migration via McClellan transformations: Geophysics, $56,1778-1785$.

Holberg, O., 1988, Toward optimum one-way wave propagation: Geophys. Prosp., 36, 99-114.

Jones, L. E. A., and Wang, H. F., 1981, Ultrasonic velocities in cretaceous shales from the Williston basin: Geophysics, 46, 288-297.

Kitchenside, P. W., 1991, Phase shift-based migration for transverse isotropy: 61 th Ann. Internat. Mtg., Soc. Expl. Geophys., Expanded Abstracts, 993-946. 
Kitchenside, P. W., 1993, 2D anisotropic migration in the space-frequency domain: Journal of seismic exploration: $2,7-22$.

Larner, K., and Cohen, J., 1993, Migration error in transversely isotropic media with linear velocity variation in depth: Geophysics, 58, 1454-1467.

Levin, F. K., 1979, Seismic velocities in transversely isotropic media: Geophysics, 44, 918-936.

Martin, D., Ehinger, A., and Rasolofosaon, P. N. J., 1992, Some aspects of seismic modeling and imaging in anisotropic media using laser ultrasonics: 62 th Ann. Internat. Mtg., Soc. Expl. Geophys., Expanded Abstracts, 1373-1376.

Nautiyal, A., Gray, S. H., Whitmore, N. D., and Garing, J. D., 1993, Stability versus accuracy for an explicit wavefield extrapolation operator: Geophysics, 58, 277283.

Postma, G. W., 1955, Wave propagation in a stratified medium: Geophysics, 20, 780-806.

Sena, A. G., and Toksöz, M. N., 1993, Kirchhoff migration and velocity analysis for converted and non-converted waves in anisotropic media: Geophysics, 58, 265-276.

Thomsen, L., 1986, Weak elastic anisotropy: Geophysics, 51, 1954-1966.

Uren, N., F., Gardner, G., H., F., and McDonald, J., A., 1990, The migrator's equation for anisotropic media: Geophysics, 55, 1429-1434.

White, J. E., Martineau-Nicoletis, L., and Monash, C., 1983, Measured anisotropy in Pierre shale: Geophys. Prosp., 31, 709-725. 


\section{LIST OF FIGURES}

Fig. 1 Detailed amplitude response in the propagation region for $\mathrm{qP}$ and $\mathrm{qSV}$ waves, obtained using the modified Taylor series (MTS) method with 39 coefficients. The normalized frequency is 0.25 cycles. $\mathrm{BS}=$ Berea sandstone, $\mathrm{MV}=$ Mesaverde clayshale, $\mathrm{TS}=$ Taylor sandstone, $\mathrm{WG}=$ weathered gypsum.

Fig. 2 Amplitude response for the same filters as in Figure 1 but now evaluated as a function of wavenumber. Wavenumbers greater than 0.25 correspond to the evanescent region.

Fig. 3 Detailed amplitude response in the propagation region for $\mathrm{qP}$ and qSV propagation, obtained using the least-squares (LS) method with 39 coefficients. The normalized frequency is 0.25 cycles.

Fig. 4 Amplitude error (left) and phase error (right) for the qP mode in the Taylor sandstone. The 39-coefficient filter was computed by the modified Taylor series method. For the amplitude error, the contour level is $1 / 1000$ for each step of depth extrapolation. The phase error contour corresponds to $\pi / 1000$ radians. Within the shaded zone, the accumulated amplitude or phase error after 1000 steps is so large that results will be inaccurate for data in that zone.

Fig. 5 Amplitude error contours (left) and phase error contours (right) for the $\mathrm{qP}$ mode in the Taylor sandstone (39-coefficient filter; least-squares method).

Fig. 6 Amplitude error for the $\mathrm{qP}$ mode in the weathered gypsum (39-coefficient filter; modified Taylor series (left) and least-squares (right) methods).

Fig. 7 Amplitude for the qSV mode in the Taylor sandstone (39-coefficient filter; modified Taylor series (left) and least-squares (right) methods).

Fig. 8 Impulse responses of migration via $\mathrm{N}=39$-coefficient explicit extrapolators for the $\mathrm{qP}$ propagation mode in the Taylor sandstone. Modified Taylor series (a) and least-squares (b) methods.

Fig. 9 Impulse responses of migration via $\mathrm{N}=39$-coefficient explicit extrapolators for the $\mathrm{qP}$ propagation mode in the weathered gypsum. Modified Taylor series (a) and least-squares (b) methods.

Fig. 10 Migration impulse responses via $\mathrm{N}=39$-coefficient, explicit extrapolators for qSV waves in the Taylor sandstone (a) and qSV waves in the Mesaverde clayshale (b). Least-squares method.

Fig. 11 Reflector model consisting of horizontal reflectors and segments with dip ranging from 30 to 90 degrees. 
Fig. 12 (a). Zero-offset synthetic section for a model with the anisotropy properties of Mesaverde clayshale, corresponding to the reflector model in Figure 11 and vertical velocity $\alpha_{0}(x, z)=3.794+0.5 x+0.5 z \mathrm{~km} / \mathrm{s}$. (b). Depthmigration obtained using a LS table of extrapolators. 

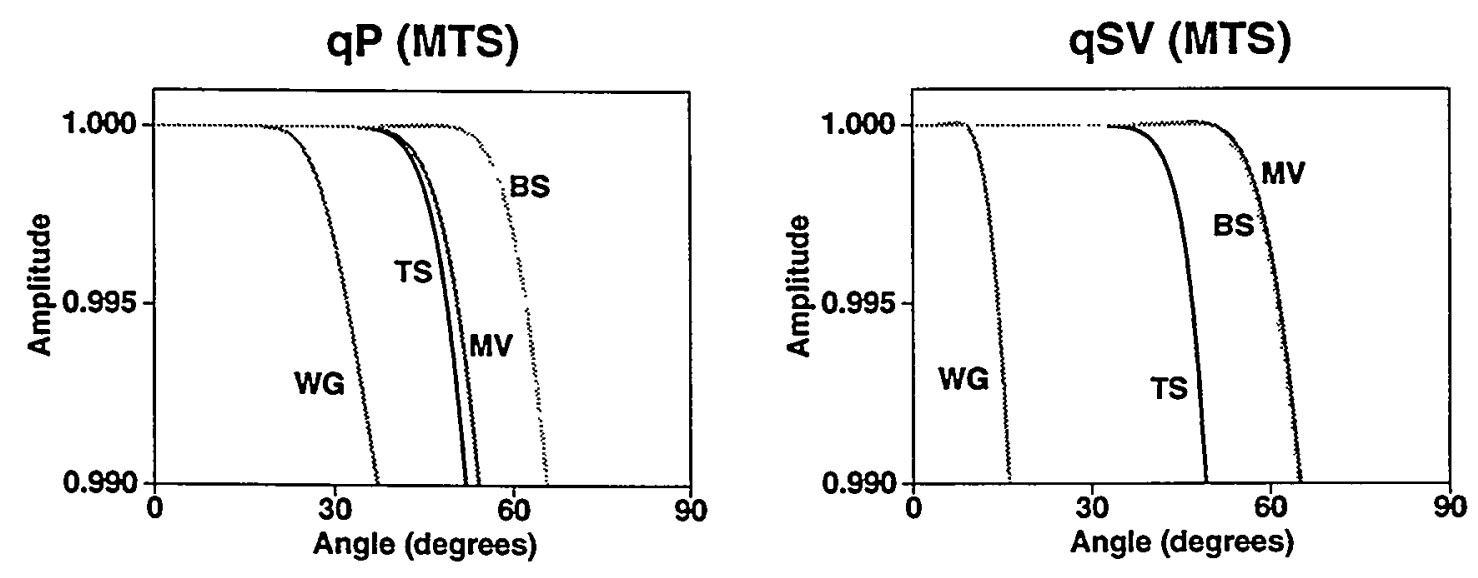

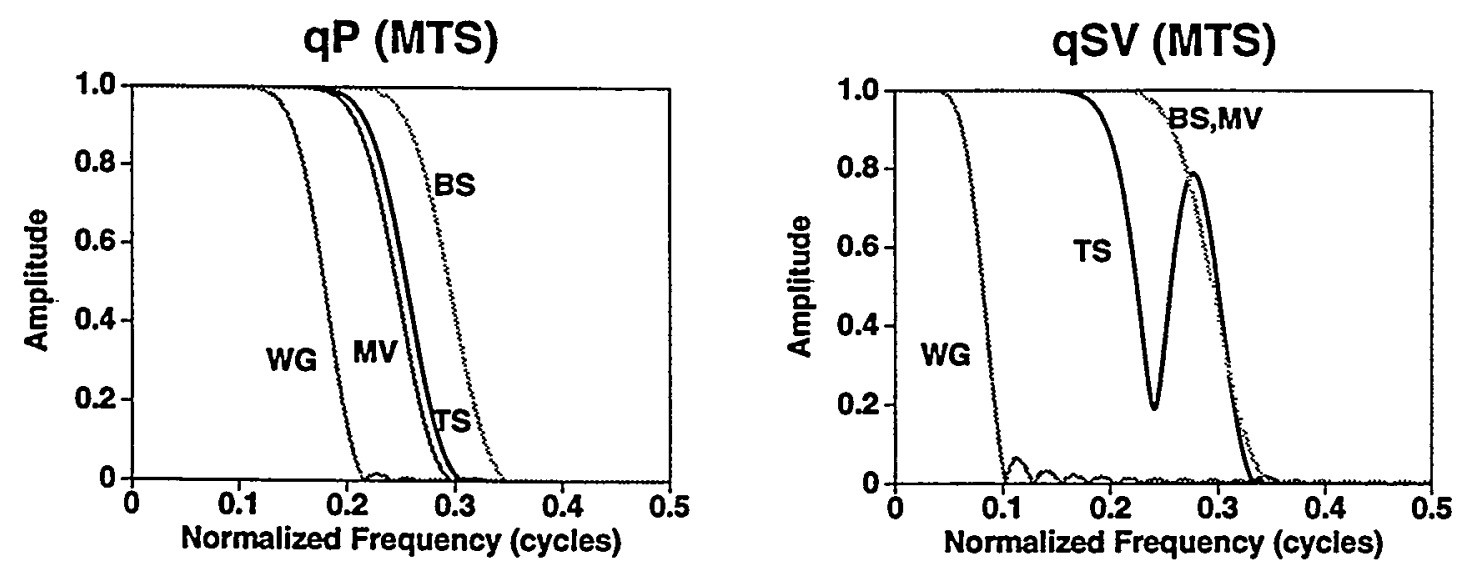

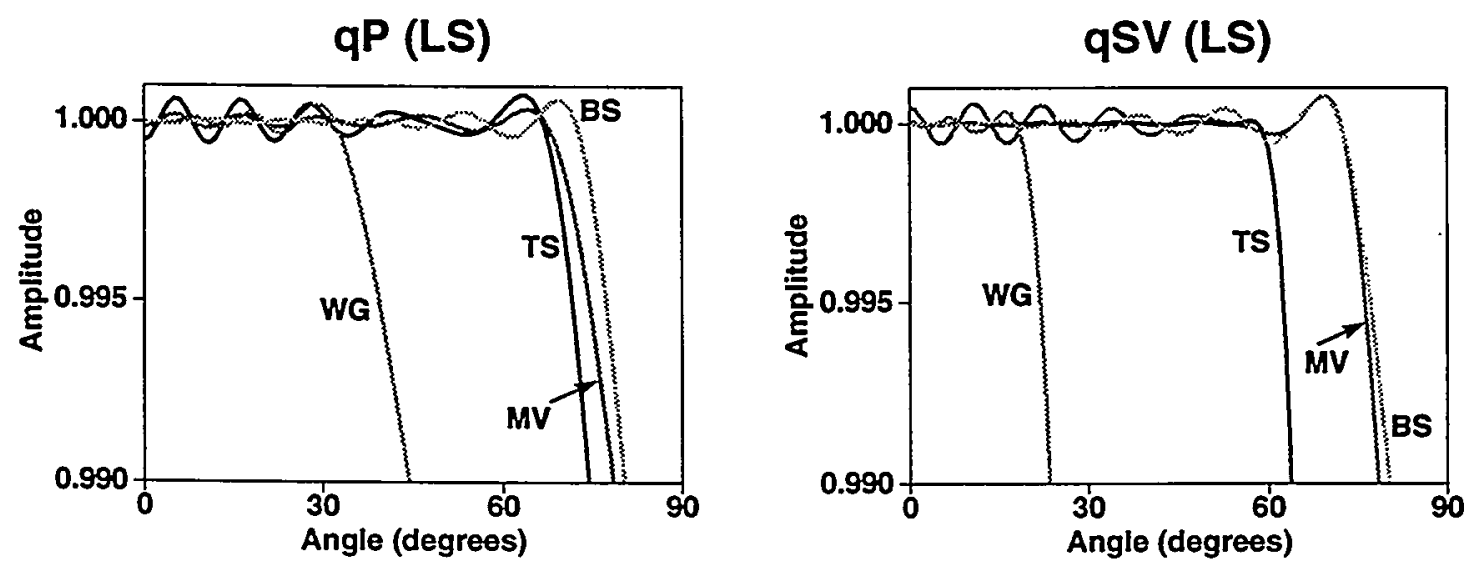

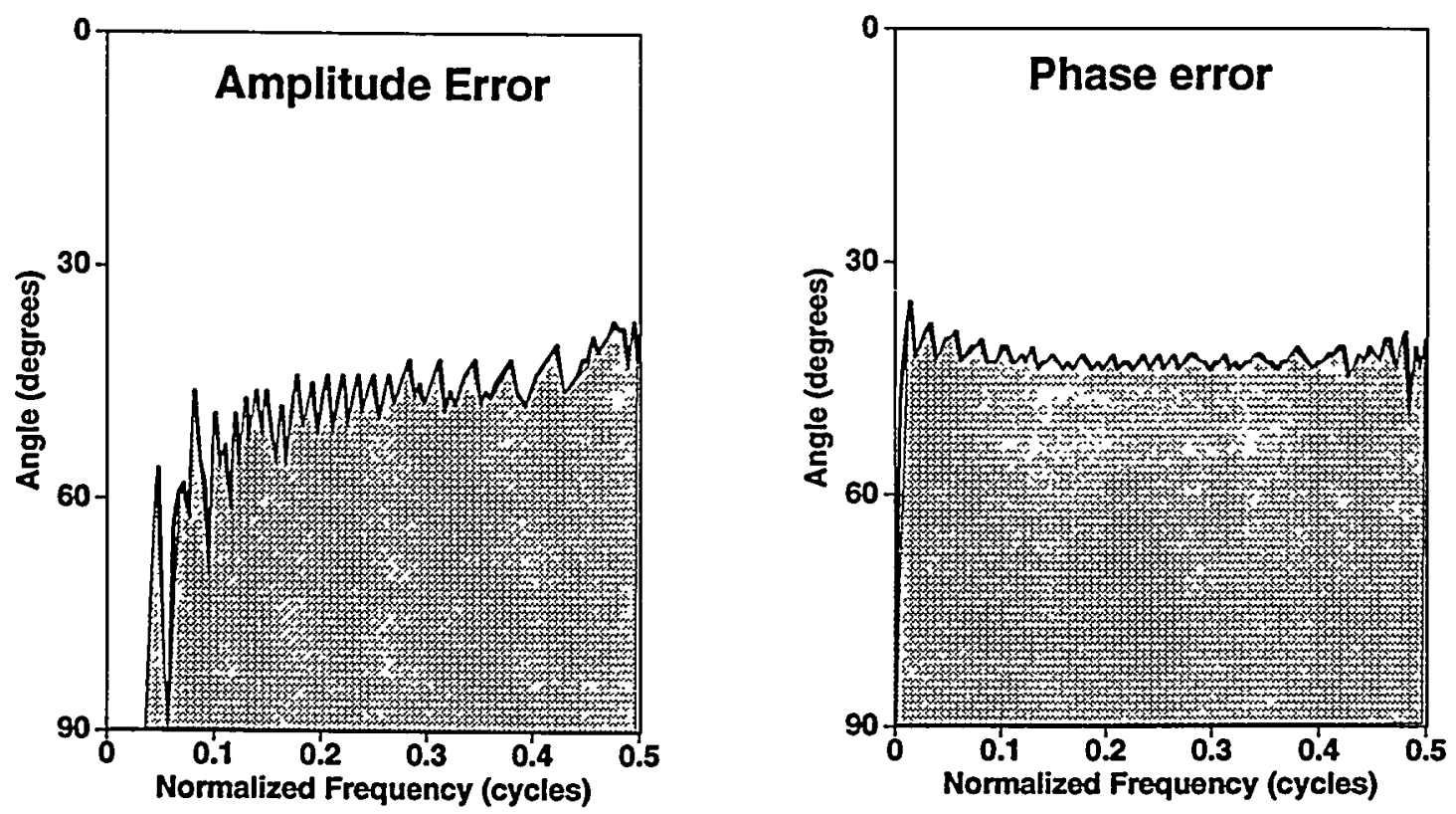

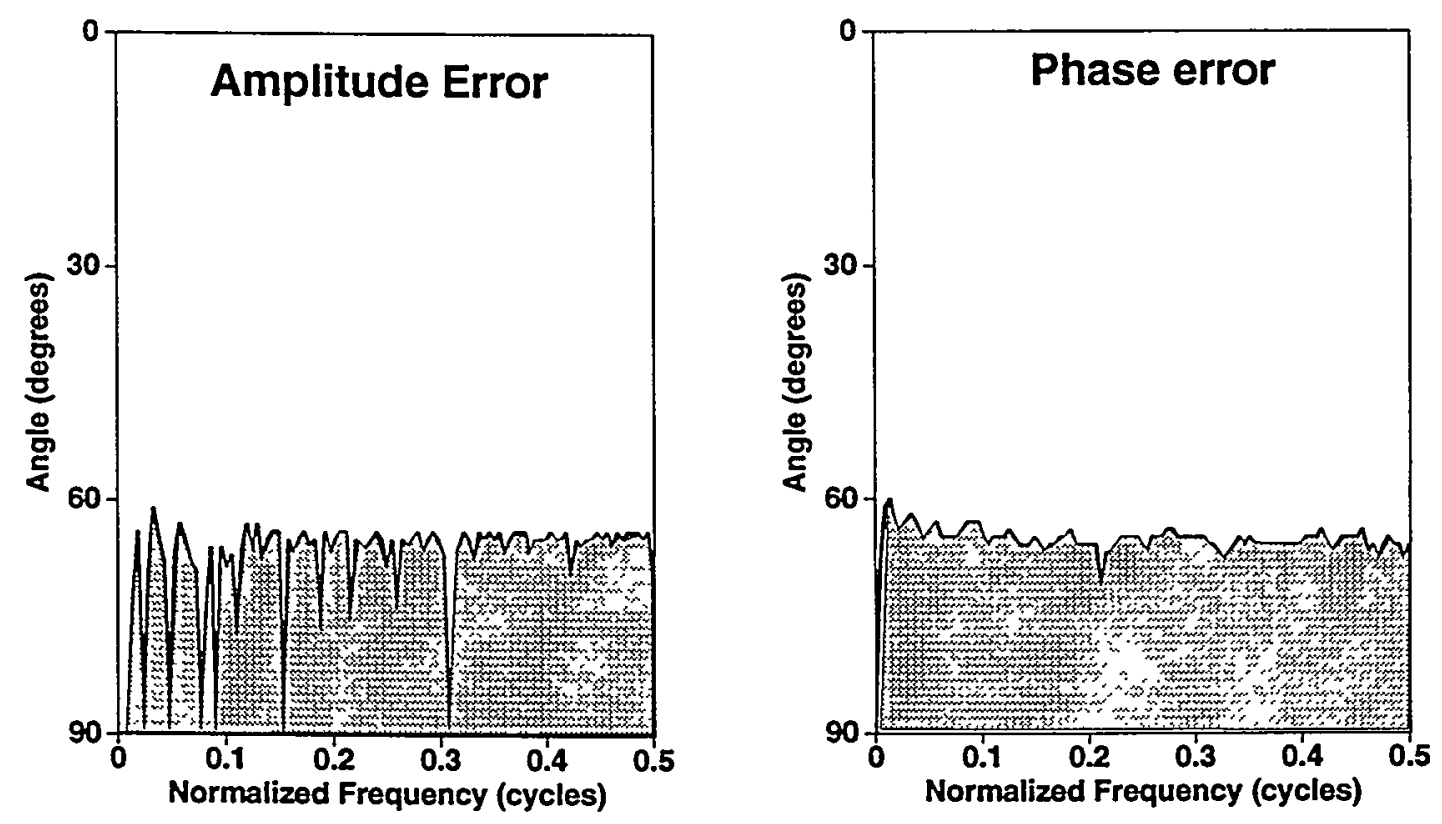

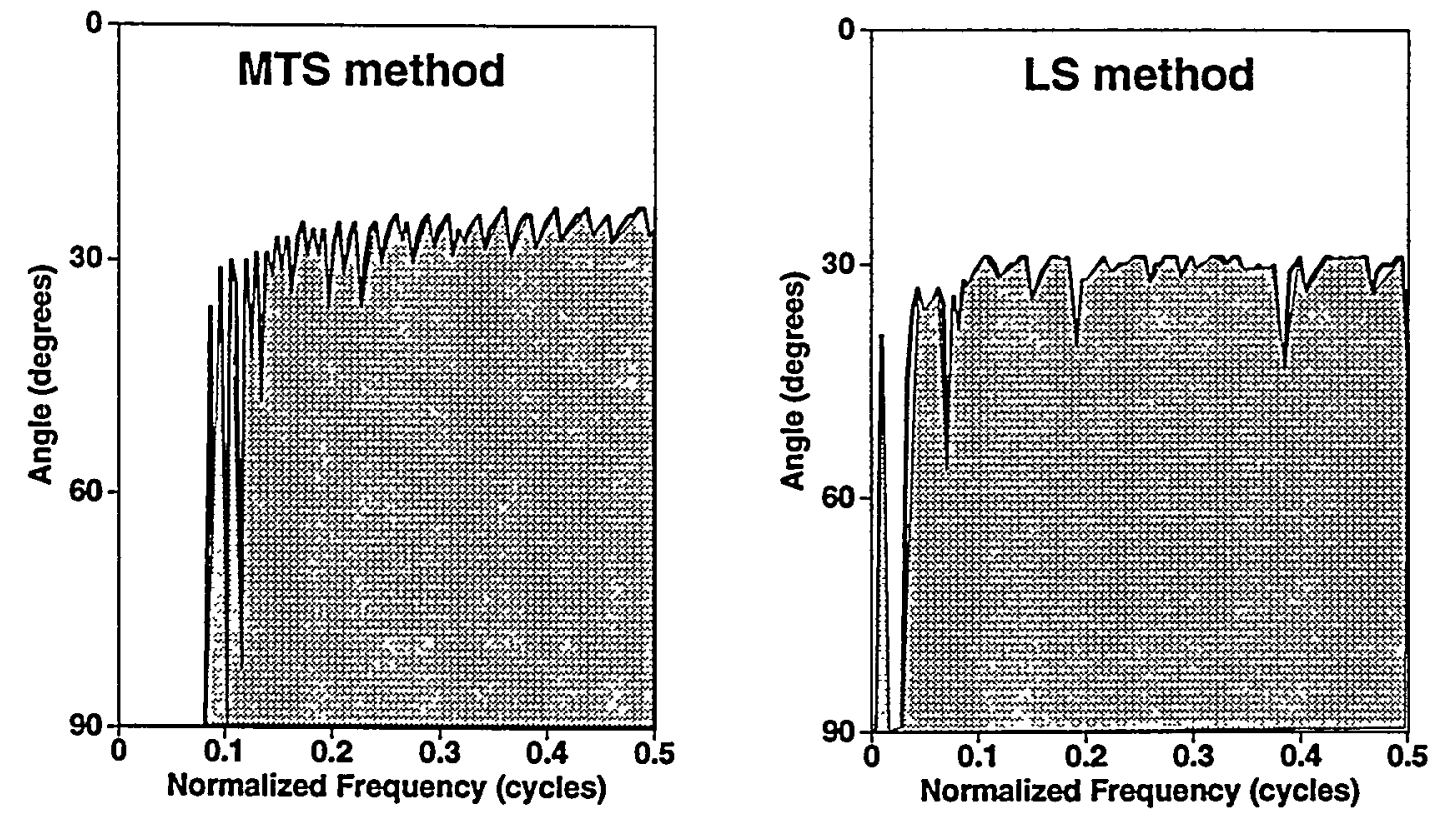

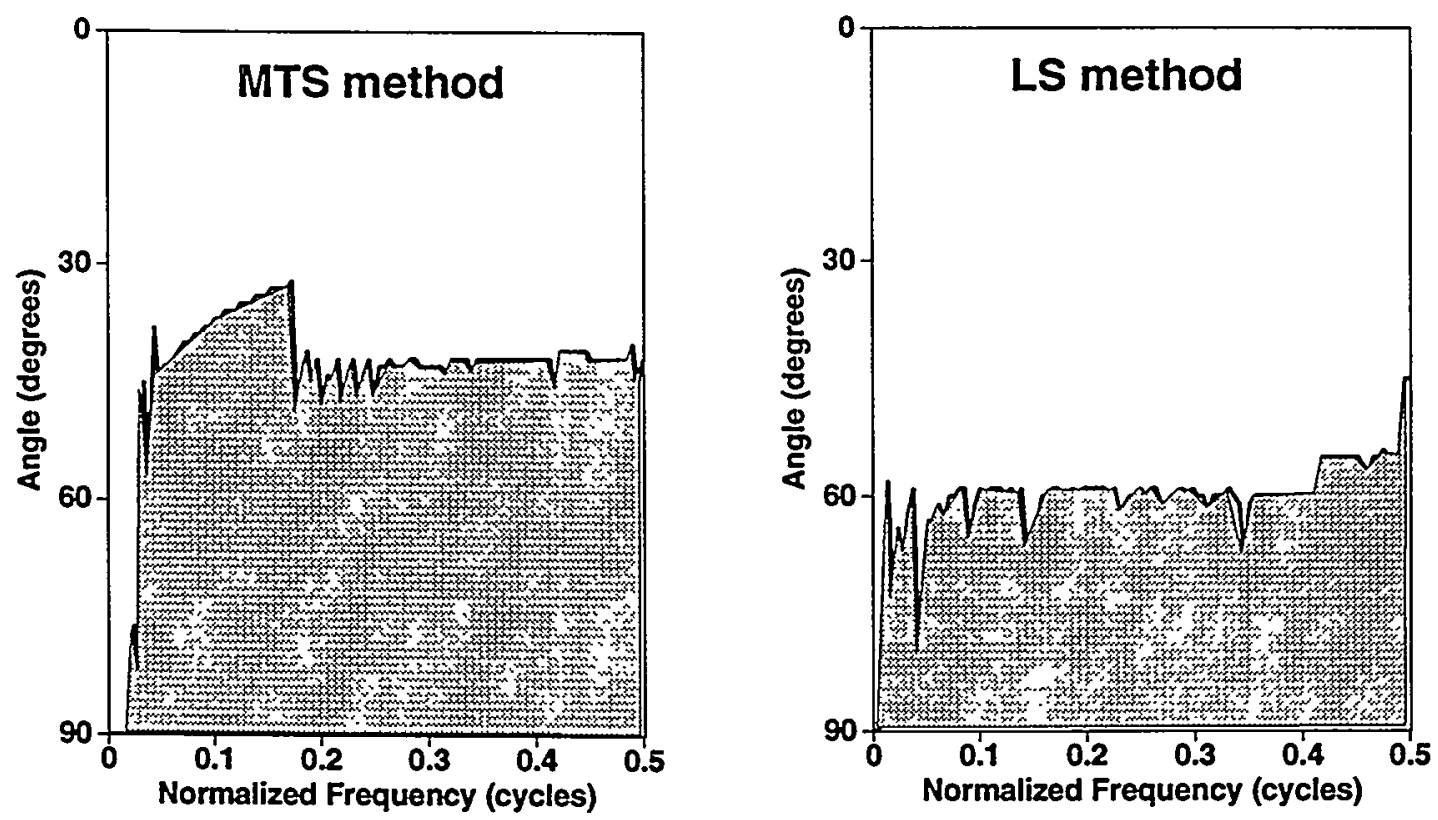

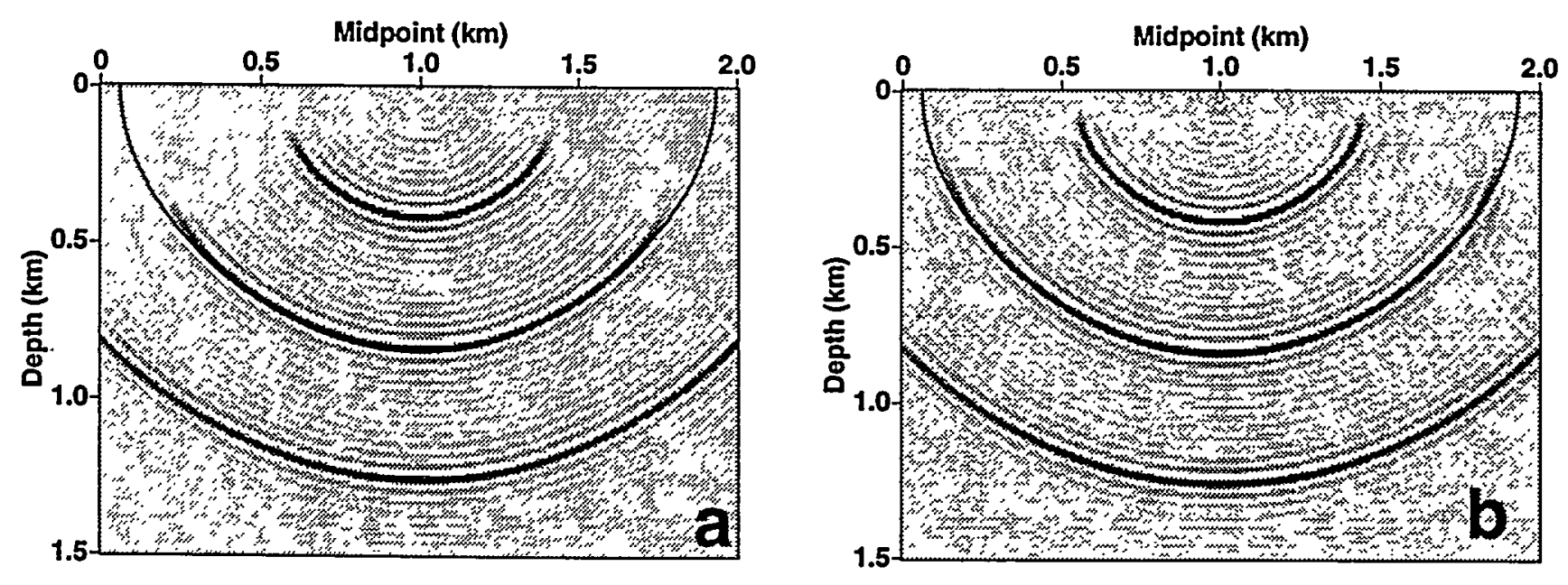

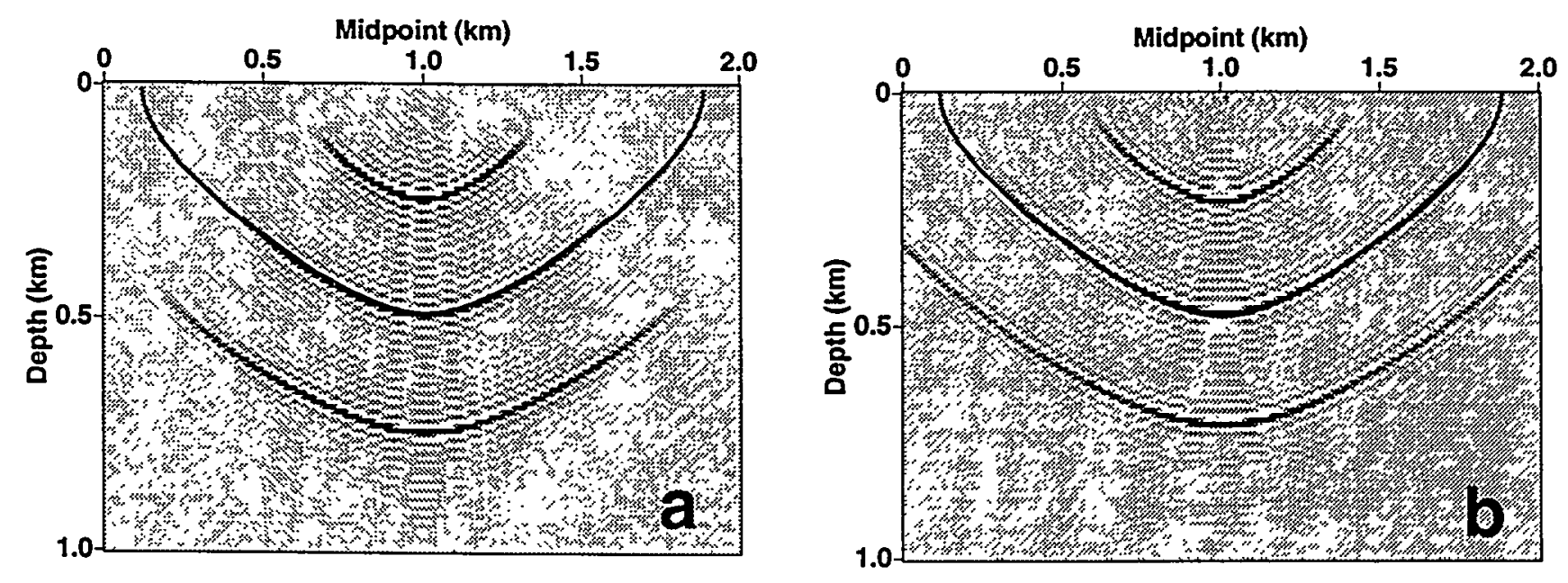

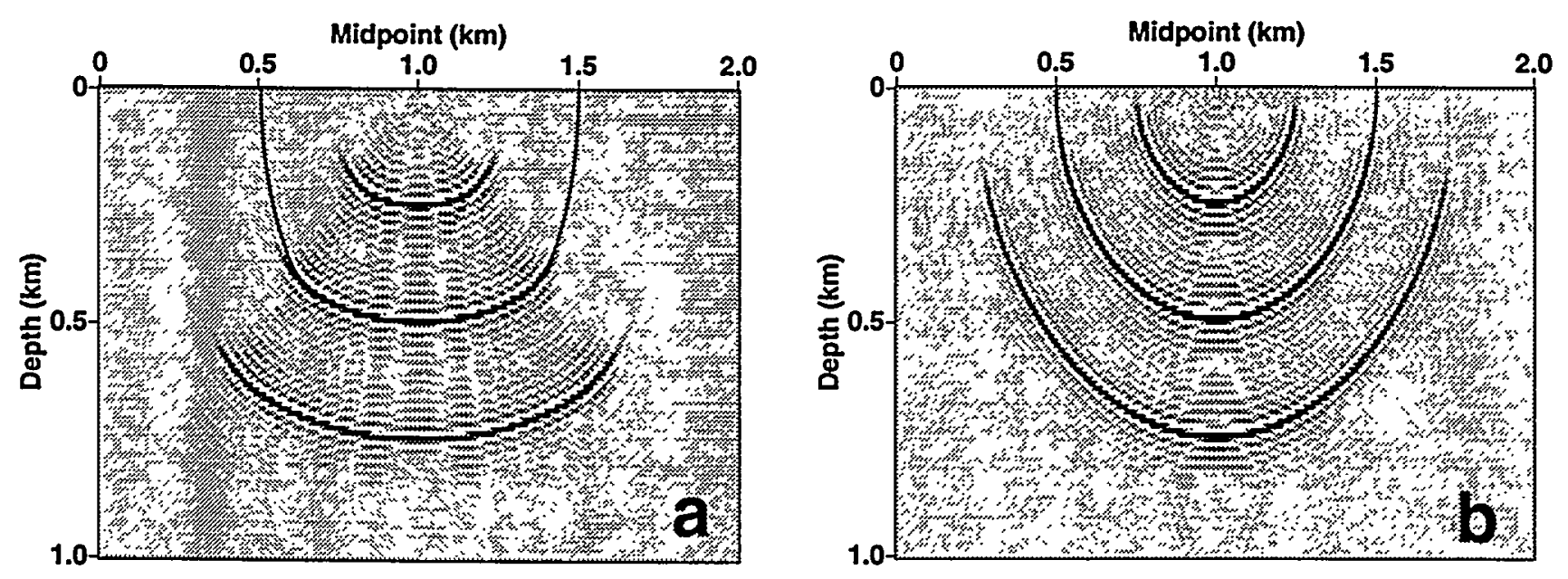


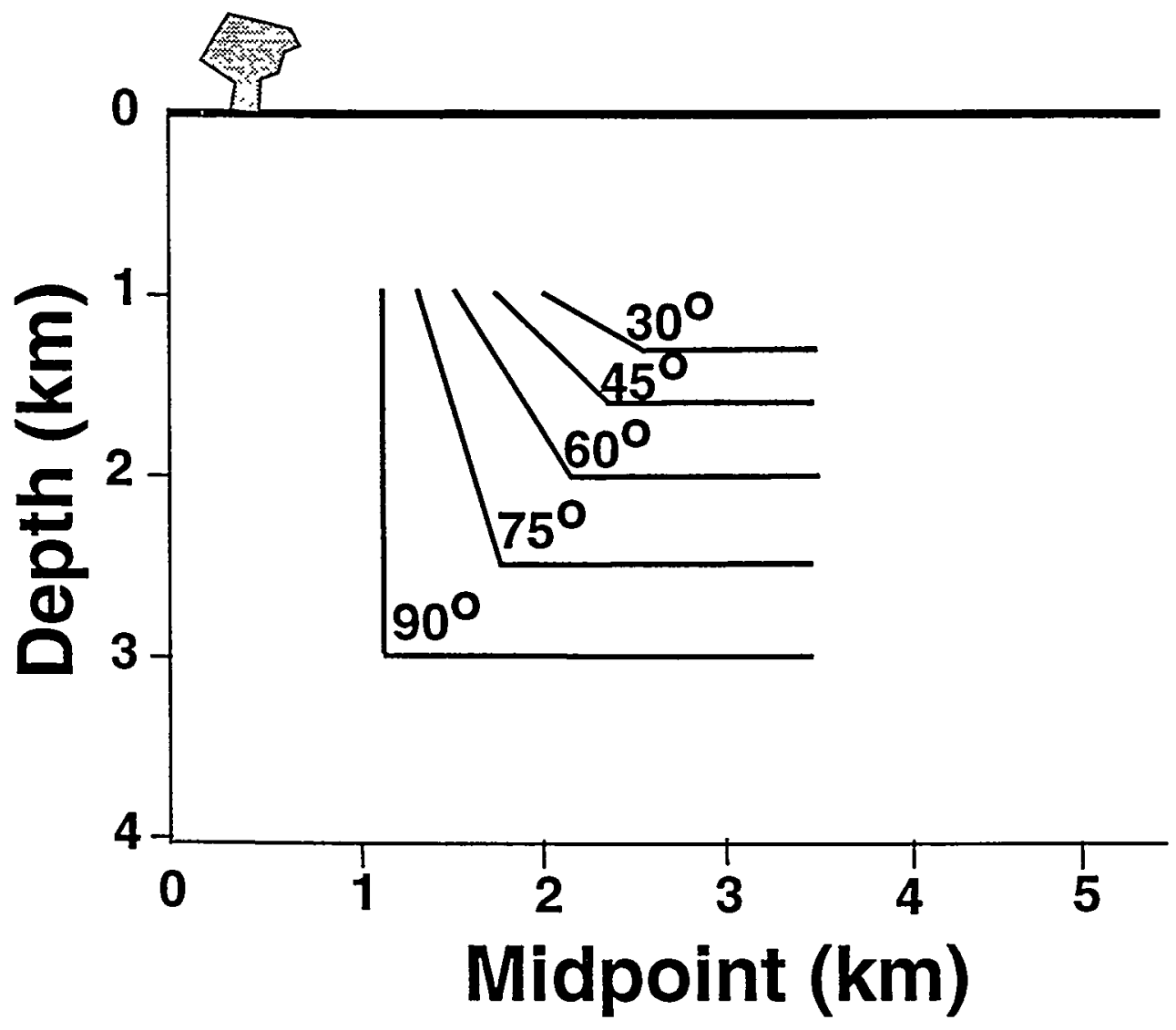



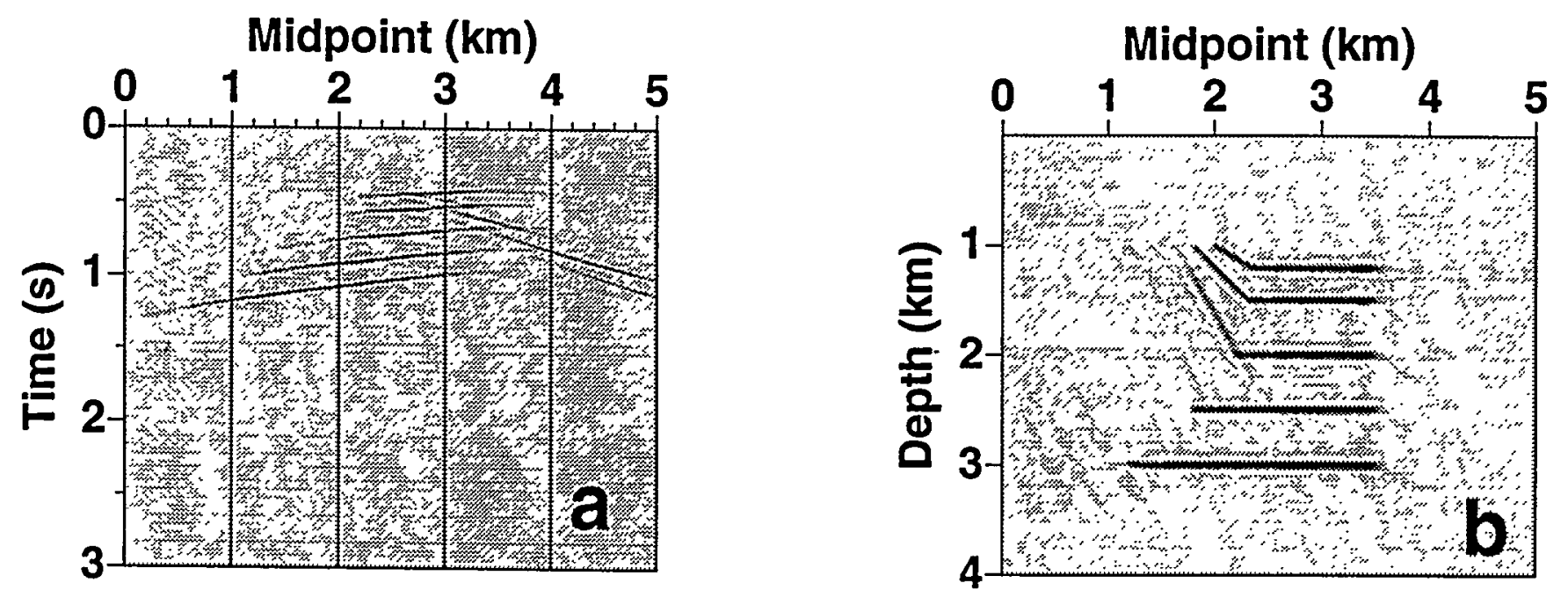